\title{
DISTRIBUTIONAL AND ENTIRE SOLUTIONS OF ORDINARY DIFFERENTIAL AND FUNCTIONAL DIFFERENTIAL EQUATIONS
}

\author{
S.M. SHAH \\ Department of Mathematics \\ University of Kentucky \\ Lexington, Kentucky 40502 \\ and \\ JOSEPH WIENER \\ Department of Mathematics \\ Pan American University \\ Edinburg, Texas 78539 \\ (Received February 25, 1983)
}

\begin{abstract}
A brief survey of recent results on distributional and entire solutions of ordinary differential equations (ODE) and functional differential equations (FDE) is given. Emphasis is made on linear equations with polynomial coefficients. Some work on generalized-function solutions of integral equations is also mentioned.
\end{abstract}

KEY WORDS AND PHRASES. Ordinary Differential Equations, Functional Differential Equations, Integral Equations, Distributional Solutions, Entire Solutions. 1980 MATHEMATICS SUBJECT CLASSIFICATION CODES. 34A20, 34A25, 34K25, 34K99, 30D05, $46 F 10,45 E 99$.

1. INTRODUCTION AND PRELIMINARIES.

This paper may be considered as a continuation of [1] which contains, in part1cular, a survey of recent results on entire solutions of ODE with polynomial coefficients. Integral transformations establish close links between entire and generalized functions [2]. Therefore, a unified approach may be used in the study of both distributional and entire solutions to some classes of linear ODE and, especially, FDE with linear transformations of the argument [3]. It is well known [4] that normal linear homogeneous systems of ODE with infinitely differentiable coefficients have no generalized-function solutions other than the classical 
solutions. In contrast to this case, for equations with singularities in the coefficients, new solutions in generalized functions may appear as well as some classical solutions may disappear. In Section 2 results on distributional and entire solutions of $O D E$ are discussed. In Section 3 we study analogous problems for FDE. Research in this direction, still developed insufficiently, discovers new aspects and properties in the theory of ODE and FDE. In fact, there are some striking dissimilarities between the behavior of ODE and FDE which deserve further investigation.

1. Distributional solutions to linear homogeneous FDE may be originated either by singularities of their coeffictents or by deviations of argument. In [5] it has been proved that the system

$$
\mathbf{x}^{\prime}(t)=\mathrm{Ax}(\mathrm{t})+\mathrm{tBx}(\lambda t),-1<\lambda<1
$$

has a solution in the class of distributions - an impossible phenomenon for ODE without singularities.

2. In [6] it was shown that a first-order algebraic ODE has no entire transcendental solutions of order less than $\frac{1}{2}$, whereas even linear first-order FDE may possess such solutions of zero order [3], [7].

3. It is well known [8] that the solution of the initial-value problem for a normal linear ODE with entire coefficients is an entire function. Let in the linear FDE

$$
w^{\prime}(z)=a(z) w(\lambda(z))+b(z), w(0)=w_{0}
$$

the functions $a(z), b(z), \lambda(z)$ be regular in the disk $|z| \leq 1$, and $\lambda(0)=0$, $|\lambda(z)| \leq 1$ for $|z| \leq 1$. Then there is a unique solution of the problem regular in $|z|<1[9]$. In general, this solution cannot be extended beyond the circle $|z|=1$, If even $a(z), b(z)$, and $\lambda(z)$ are entire functions. Thus, the solution of the equation $w^{\prime}(z)=a(z) w\left(z^{2}\right)$, where $a(z)$ is an entire function with positive coefficients, has the circle $|z|=1$ as the natural boundary [10], [11] .

2. DISTRIBUTIONAL AND ENTIRE SOLUTIONS OF ODE

The number $m$ is called the order of the distribution 


$$
x=\sum_{k=0}^{m} x_{k} \delta^{(k)}(t), x_{m} \neq 0,
$$

where $\delta^{(k)}$ denotes the kth derivative of the Dirac $\delta$ measure, and the variable $t$ is real. Finite order solutions of linear ODE have been studied mainly for equations with regular singular points [12 - 16]. In [16] for the first time an existence criterion of solutions (2.1) to any linear ODE was established.

THEOREM 2.1. (Wiener [16]). If the equation

$$
\sum_{i=0}^{n} a_{i}(t) x^{(n-i)}(t)=0
$$

with coefficients $a_{i}(t) \varepsilon c^{(m+n-1)}$ in a neighborhood of $t=0$ has a solution of order m concentrated on $t=0$, then:

(1) $a_{0}(0)=0$,

(2) m satisfies the relation $-(m+n) a_{0}^{\prime}(0)+a_{1}(0)=0$,

(3) there exists a nontrivial solution $\left(x_{0}, \ldots, x_{m}\right)$ of the system

$$
\begin{gathered}
\sum_{j=0}^{m+n} x_{k+j-n} \sum_{i=0}^{\min (j, n)}(-1)^{j-i} a_{i}^{(j-i)}(0)(k+j-i) !=0 \\
(k=0,1, \ldots, m+n) .
\end{gathered}
$$

THEOREM 2.2. (Wiener [16]). Eq. (2.2) has an $m$ order solution with support $t=0$, if the following hypotheses are satisfied:

(i) For some natural $N(0 \leq N \leq m+n)$,

$$
a_{i}^{(N-i)}(0)=0, i=0, \ldots, \min (N, n) ;
$$

(ii) $m$ is the smallest nonnegative integer root of the relation

$$
\sum_{i=0}^{M}(-1)^{N+1-i} a_{j}^{(N+1-i)}(0)(m+n-i) !=0, M=\min (N+1, n),
$$

where $\mathrm{N}$ denotes the greatest number for which (i) holds;

(iii) there exists a nonzero solution of system (3) in Th. 2.1.

From these theorems it follows that if the equation

$$
\sum_{i=0}^{n} t^{i} a_{i}(t) x^{(i)}(t)=0
$$

with coefficients $a_{i}(t) \varepsilon C^{m}$ and $a_{n}(0) \neq 0$ has a solution $(2.1)$ of order $m$, then 


$$
\sum_{i=0}^{n}(-1)^{i} a_{i}(0)(m+i) !=0 .
$$

Conversely, if $\mathrm{m}$ is the smallest nonnegative integer root of (2.4), there exists an $m$ order solution of (2.3) concentrated on $t=0[16]$. This proposition constitutes the basis for the study of finite order solutions to equations with regular singular points. The stated results can be used also in the search of polynomial and rational solutions to linear ODE with polynomial coefficients.

Thus, we formulate

THEOREM 2.3. The equation

$$
\sum_{i=0}^{n}\left(a_{1} t+b_{1}\right) x^{(n-1)}(t)=0
$$

with constant coefficients $a_{i}, b_{i}$ and $a_{0}=1, b_{0}=0$ has a finite order solution if all poles $s_{i}$ of the function

$$
R(s)=\sum_{i=0}^{n}\left(b_{i} s-(n-i) a_{i}\right) s^{n-i-1} / \sum_{i=0}^{n} a_{i} s^{n-i}
$$

are real distinct and all residues $r_{i}=\underset{s=s}{\text { res }} R(s)$ are nonnegative integral.

This solution is given by the formula

$$
x=c \prod_{I=1}^{n}\left(d / d t-s_{f}\right)^{r} i_{\delta(t), c}=\text { const }
$$

and its order is

$$
m=\sum_{i=1}^{n} r_{i} \text {. }
$$

If $a_{n}=0$ there exists also a solution

$$
x=c \prod_{i=1}^{n}\left(d / d t-s_{i}\right)^{r} t^{-1} \text {. }
$$

Polynomial and rational solutions of ODE have been studied extensively [17 -

25]. In [17] the author deals with the equation

$$
\sum_{i=0}^{n} a_{1} x^{(t)}(t)=0
$$

where $a_{f}=a_{1}(t)$ have mth order derivatives in $[a, b]$. Let $a_{10}=a_{1} / a_{0}$ 
(provided $a_{0} \neq 0$ ) and $a_{i j}=\left(a_{i+1, j-1}^{\prime}+a_{i, j-1}\right) /\left(a_{i, j-1}^{\prime}+a_{0, j-1}\right)$

(provided $a_{1, j-1}^{i}+a_{0, j-1} \neq 0$ ).

THEOREM 2.4. (Šapkarev [17]). Eq. (2.5) has a polynomial solution of degree $m$ if and only if

$$
a_{1, m-1}^{1}+a_{0, m-1}=0 \text {. }
$$

THEOREM 2.5. (Yapkarev [17]). Eq. (2.5) has polynomial solutions of degrees m, $m+1, \ldots, m+n-1$ if and only if

$$
a_{i+1, m-1}^{\prime}+a_{i, m-1}=0 \text { for } i=0, I, \ldots, n-1 \text {. }
$$

Necessary and sufficient conditions for the existence of a maximal number of polynomial solutions to algebraic differential equations are given in [18] and [19]. Existence of polynomial solutions of an equation of Liénard type is studied in [20]. The equation $w^{\prime}=a_{0}(z)+a_{1}(z) w+\ldots+a_{n}(z) w^{n}$ with $n$ polynomials as solutions is considered in [21]. In a number of papers additions to Kamke's treatise are made. Thus, in [22] it is proved that for the generalization

$$
x=\sum_{i=1}^{n}(-1)^{i-1} t^{i} x^{(i)} / i !+f\left(x^{(n)}\right)
$$

of Clairaut's equation the following are two solutions:

$$
x=\sum_{i=1}^{n} c_{i} t^{i} / i !+f\left(c_{n}\right),
$$

where $c_{1}, \ldots, c_{n}$ are arbitrary constants, and

$$
x=y+\sum_{i=1}^{n-1} A_{i} t^{i}
$$

where $A_{1}, \ldots, A_{n-1}$ are arbitrary constants and $f^{\prime}\left(y^{(n)}\right)=(-t)^{n} / n !$. In [23] and [24] rational solutions of Painlevé's third and fifth equations are studied.

THEOREM 2.6. (Gromak, [23]). There exists a rational solution of the equation

$$
z w w^{\prime \prime}=z w^{\prime 2}-w w^{\prime}+\alpha w^{3}+\beta w+\gamma z w^{4}+\delta z,
$$

where $\alpha \delta \neq 0, \gamma=0$ or $\beta \gamma \neq 0, \delta=0$, if $\beta=2 k, k=0, \pm 1, \pm 2, \ldots$; this rational solution has $\beta^{2} / 4$ poles and $\frac{\beta^{2}}{4}+1$ zeros.

The work [25] concerns the study of properties of solutions to the complex equation (1) $\mathrm{Pf}=\mathrm{g}$, where 


$$
P(z, \partial / \partial z)=\sum_{k=0}^{m} P_{k}(z) \partial^{k} / \partial z^{k}, \partial / \partial z=\frac{1}{2}(\partial / \partial x-1 \partial / \partial y)
$$

and $\mathrm{g}$ is a given holomorphic or rational function. Various conditions guaranteeing that the solutions of (1) are polynomial or rational functions of a certain type are obtained. In the last part, differential equations of Euler type are considered.

THEROEM 2.7. (Nova[25]). Let $\Omega$ be a simply connected open set in $\phi$ and $a \varepsilon \Omega$. If $a$ is a regular singular point of $P$ and every solution of $P f=g$ in $O(\Omega \backslash\{a\})$, with $g \in R_{a}(\Omega)$, is rational in $\Omega$ with a pole at $a$, then $P$ is normal.

Significant contributions to the study of asymptotic properties of the analytic solutions of algebrafc ordinary and partial differential equations are made in [6]. The main properties are the growth of an entire solution, the order of a meromorphic solution and its exceptional values. In a certain sense, this book completes the fundamental monograph [26]. In the second chapter of [6], the author studies the algebraic DE

$$
P\left(z, w, w^{\prime}\right)=0 .
$$

It is reduced to the form

$$
P_{0}\left(z, w, \frac{z w^{\prime}}{w}\right) \equiv \sum_{i=0}^{n} Q_{i}\left(z, \frac{z w^{\prime}}{w}\right) w^{n-1}=0,
$$

where $Q_{1}(z, \eta)$ are polynomials in $z$ and $\eta$. Let $w(z)$ be an entire transcendental solution of (2.6) and let $\zeta$ be a point on the circle $|z|=r$ such that

$$
|w(\zeta)|=M(r)=\max |w(z)|,|z|=r
$$

Substituting $w=w(z), z=\zeta$ in $(2.7)$ and dividing its terms by $w^{n}(\zeta)$ gives, with regard to Macintyre's formula [27]

$$
\zeta f^{\prime}(\zeta) / f(\zeta)=r M^{\prime}(r) / M(r)=K(r),
$$

the equation

$$
Q_{0}(\zeta, K(r))=-\sum_{i=1}^{n} Q_{1}(\zeta, K(r)) w^{-1}(\zeta)
$$

From here it follows that

$$
\mathrm{Q}_{0}(\zeta, \mathrm{K}(\mathrm{r}))=\mathrm{o}(1) \text {. }
$$

The polynomial $Q_{0}(\zeta, K)$ is called the principal polynomial of Eq. (2.7), and (2.8) is called the determining equation. 
THEOREM 2.8. (Strelitz[6]). The order and type of an entire transcendental solution of $(2.6)$ are equal, respectively, to the positive order $\rho_{j}>0$ and type $\sigma_{j}$ of one of the solutions of the determining equation (2.8). Furthermore,

$$
\lim _{r \rightarrow \infty} K(r) / r^{\rho_{j}}=\sigma_{j} \rho_{j}, \lim _{r \rightarrow \infty} \ln M(r) / r^{\rho_{j}}=\sigma_{j} .
$$

The following proposition shows that not all of the numbers $\rho_{1}$ indicated in Th. 2.8 may be the orders of the entire solutions of first order algebraic DE.

THEOREM 2.9. (Strelitz[6]). Algebraic DE(2.6) cannot have entire transcendental solutions of order $\rho<\frac{1}{2}$. In general, $\frac{1}{2}$ cannot be replaced by a larger number: there are equations of the form (2.6) that have entire transcendental solutions of order $\frac{1}{2}$.

EXAMPLE 2.1. (Strelitz[6]). The equation

$$
w^{2}+4 z w^{2}=1
$$

has an entire transcendental solution $w=\cos \sqrt{2}$ of order $\rho=\frac{1}{2}$.

The following result is of interest in this connection.

THEOREM 2.10. (Wittich [26]). Let $R(z, w)$ be a rational function of $z$ and $w$. A meromorphic solution of the equation $w^{\prime}=R(z, w)$ which is of order $<\frac{1}{2}$ is a rational function.

In the second chapter of [6] it is also proved that the order of any meromorphic solution of a first order algebraic DE is finite. The orders of the transcendental entire solutions of second order linear DE with polynomial coefficients have been investigated in [28], [29], [30]. Suppose that $P(z)$ and $Q(z)$ are polynomials of degree $p$ and $q$, respectively. Set $g_{0}=1+\max \left(p, \frac{1}{2} q\right)$. Let $p \geq q+1$. Then all transcendental solutions of the equation

$$
w^{\prime \prime}+P(z) w^{\prime}+Q(z) w=0
$$

are of the order $1+p=g_{0}$. If $p \leq \frac{1}{2} q$, all transcendental solutions are of the order $1+\frac{1}{2} q=g_{0}$. Deviation from this pattern can occur only if $\frac{1}{2} q<p \leq q$. Here $g_{0}=1+p$, and there are always solutions of this order; under certain circumstances, however, a lower order $q-p+1$ may also be present.

THEOREM 2.11. (Hille [30]). If in (2.9) either $P$ or $Q$ is an entire 
transcendental function while the other is a polynomial, then every transcendental solution of (2.9) is an entire function of infinite order. This is not necessarily true, however, if both $P$ and $Q$ are entire.

THEOREM 2.12. (Wittich [30]). In (2.9) suppose that $P$ and $Q$ are entire functions and suppose that the equation has a fundamental system $w_{1}(z), w_{2}(z)$, where $w_{1}$ and $w_{2}$ are entire functions of order $\rho_{1}$ and $\rho_{2}$, respectively. Then $P$ and $Q$ are polynomials.

Th. 2.12 may be regarded as a converse of Th. 2.11.

THEOREM 2.13. (Frei [31]). Suppose that in the equation

$$
w^{(n)}+\sum_{i=1}^{n} p_{i}(z) w^{(n-i)}=0
$$

the coefficients $p_{i}(z)(i=1,2, \ldots, k)$ are polynomials, and $p_{k+1}(z)$ is an entire transcendental function. Under these conditions the equation can have no more than $k$ linear independent entire transcendental solutions of finite order, whereas all other solutions of the fundamental system are of infinite order.

The results by Frei, Pösch1, and Wittich on the growth of solutions of linear $D E$ are generalized in the third chapter of [6]. The main tool is the Wiman-Valiron method, but the case when this method fails is also studied. Nonlinear algebraic $D E$ of the form $P\left(z, w, w^{\prime}, \ldots, w^{(n)}\right)=0$ are investigated, too. A necessary condition for some complex number $a$ to be a defect value of a meromorphic solution of finite order is $\mathrm{P}(\mathrm{z}, a, 0, \ldots, 0) \equiv 0$. We already know that first order algebraic DE have no entire transcendental solutions of zero order. In [32] it is shown that there are algebraic DE of third order that have entire transcendental solutions of zero order.

THEOREM 2.14. (Zimogiiad [33]). A second order algebraic differential equation

$$
P\left(z, w, w^{\prime}, w^{\prime \prime}\right)=0
$$

( $P$ is a polynomial of all its variables) cannot have entire transcendental solutions of zero order.

THEOREM 2.15. (Shah [34]). Let $f(z)$ be an entire solution of an $n$th order linear homogeneous equation 


$$
P_{0}(z) w(n)+\ldots+P_{n}(z) w(z)=0
$$

where $P_{j}(0<j \leq n)$ are all polynomials. Write $\underset{0<j \leq n}{\max } \operatorname{deg} P_{j}=d$ and suppose that $\operatorname{deg} P_{0}=\operatorname{deg} P_{n}>\max \operatorname{deg} P_{j},(1 \leq j \leq n-1)$. Then $f(z)$ is of order 1 and exponential type $\mathrm{T}$ where

$$
T=\left|a_{n} / a_{0}\right|^{1 / n}
$$

and $a_{p}=\lim _{z \rightarrow \infty} P_{p}(z) / z^{d}, p=0, n$. For cases when the condition on the degree of $P_{f}$ is not satisfied, see ([34, Th. 1.6]).

The Bessel function of integer order $n, J_{n}(z)$, satisfies the ODE $z^{2} w^{\prime \prime}+z w^{\prime}+\left(z^{2}-n^{2}\right) w=0$, and the Coulomb wave function $F_{L}(n, z)$ satisfies the ODE $z^{2} w^{\prime \prime}+\left(z^{2}-2 n z-L(L+1)\right) w=0(n$ a real constant, L a nonnegative integer). For these functions we have $\log M\left(r, J_{n}\right) \sim r \sim \log M\left(r, F_{L}\right)$ as $r \rightarrow \infty$.

Consider now vector-valued functions $F: \phi^{1} \rightarrow k^{m}$. Suppose that the components $f_{k}(1 \leq k \leq m)$ are all entire functions. Write

$$
\|F(z)\|=\max \left\{\left|f_{k}(z)\right|, 1 \leq k \leq m\right\}, M(r, F)=\max _{|z|=r}\|F(z)\| .
$$

DEFINITION. A vector-valued entire function $F$ is said to be of bounded index (BI) if there exists an integer $\mathrm{N}$ such that

$$
\max _{0 \leq i \leq N} \frac{\left\|F^{(i)}(z)\right\|}{i !} \geq \frac{\left\|F^{(k)}(z)\right\|}{k !}
$$

for all $z \varepsilon \phi^{1}$ and $k=0,1, \ldots$ The least such integer $N$ is called the index of $F$. THEOREM 2.16. (Roy and Shah [35]). Let F: $k^{1} \rightarrow k^{m}$ be a vector-valued entire function of BIN. Then

$$
\|F(z)\| \leq A \exp ((N+1)|z|)
$$

where $A=\max _{0 \leq k \leq N} \frac{\left\|_{F}^{(k)}(0)\right\|}{(N+1)^{k}}$. The result is sharp.

The function $F$ may be of BI but the components $f_{k}$ may not be of BI. In the next theorem, it is shown that if $F$ satisfies an ODE then $F$ and each $f_{k}$ are of BI. Let $R$ denote the class of all rational functions $r(z)$ bounded at infinity and $Q_{i}(z)$ $(1 \leq 1 \leq \mathrm{m})$ denote an $\mathrm{m} \times \mathrm{m}$ matrix with entries in $\mathrm{R}$. Write 
and

$$
Q_{i}(z)=\left(a_{p q, i}(z)\right), \lim _{z \rightarrow \infty}\left|a_{p q, i}(z)\right|=\left|A_{p q, i}\right|
$$

$$
\sup \left(\left|A_{p q, i}\right|, 1 \leq p, q \leq m\right)=\left|A_{i}\right| .
$$

THEOREM 2.17. (Roy and Shah [35]). Let $F: \phi^{1} \rightarrow \boldsymbol{k}^{\mathrm{m}}$ be a vertor-valued function whose components $f_{1}, \ldots, f_{m}$ are all entire functions. Suppose that $F$ satisfies the ODE

$$
L_{n}(w, z, Q) \equiv w^{(n)}(z)+Q_{1}(z) v^{(n-1)}(z)+\ldots+Q_{n}(z) w(z)=g(z)
$$

where $g(z)$ is a vector-valued entire function of BI. Then each $f_{k}$ satisfies an ODE of this form (with possibly different $n$ and coefficients), and $F, f_{1}, \ldots, f_{m}$ are all of $B I$. If the entries of $Q_{i}$ are not in $R$ then $F$ may not be of $B I$.

THEOREM 2.18. (Roy and Shah [35]). Let $w(z) \neq 0$ be a vector-valued entire function satisfying the ODE

$$
L_{n}(w, z, Q)=0 \text {. }
$$

Then we have:

$$
\text { (i) } \lim _{r \rightarrow \infty} \sup \frac{\log M(r, w)}{r} \leq \max \left\{1, m \sum_{i=1}^{n}\left|A_{i}\right|\right\}
$$

where the numbers $A_{i}$ are defined above.

(ii) If the elements of $Q_{1}(1 \leq i \leq m)$ are constant, and $P \geq 0$ is any integer such that

$$
m\left[\frac{\left|A_{1}\right|}{n+p}+\frac{\left|A_{2}\right|}{(n+p)(n+p-1)}+\ldots+\frac{\left|A_{n}\right|}{(n+p) \ldots(p+1)}\right] \leq 1,
$$

then the index $N$, of $F(z)$, is less than or equal to $n+p-1$. The bound on $N$ is best possible.

Next we compare these growth results with the corresponding ones for solutions of algebraic difference equations.

THEOREM 2.19. (Shah [36]). Let $P(t, u, v)$ be a polynomial with real coefficients. Let $u(t)$ be a real continuous solution of a first order algebraic difference equation $P(t, u(t), u(t+1))=0$ for $t \geq t_{0}$. Then there exists a positive number A which depends only on the polynomial $P$ such that

$$
\lim _{t \rightarrow+\infty} \inf \frac{|u(t)|}{e_{2}(A t)}=0 \text {. }
$$


If $u(t)$ is monotonic for $t \geq t_{0}$, then

$$
\lim _{t \rightarrow+\infty} \frac{|u(t)|}{e_{2}(\text { At })}=0 \text {. }
$$

Here $e_{2}(x)$ denotes $\exp (\exp x)$.

The function $e_{2}(\mathrm{At})$ cannot be replaced by a function of slower rate of 8 rowth, in general.

THEOREM 2.20. (Shah [36]). Let $\phi(t)$ be an arbitrary increasing function which tends to $+\infty$ as $t \rightarrow+\infty$. There exists an equation $P(t, u(t), u(t+1))=0$ with a real solution $u(t)$ which is continuous for $t \geq t_{0}$ and which exceeds $\phi(t)$ at each point of a sequence $\left\{t_{n}\right\}$ such that $t_{n} \rightarrow+\infty$ as $n \rightarrow \infty$.

For further results see [37], [38], [39] and [40].

Let $f(z)$ be an entire transcendental function. The $(\alpha, x)$ index is defined as $J\left(x^{\alpha}, f\right)=\max |\zeta|=r^{\nu} \zeta^{\left(x r^{\alpha}, f\right)}$

where $\nu_{\zeta}(r, f)$ is the central index of the Taylor expansion

$$
f(z)=\sum_{i=0}^{\infty} f^{(i)}(\zeta)(z-\zeta)^{i} / i !
$$

The author of [41] evaluates the $(\alpha, x)$ indices of entire transcendental solutions of linear ODE with polynomial coefficients. On the basis of these results some theorems concerning the distribution of values of these solutions are proved.

THL order $\rho$ and type $\sigma$ of an ordinary linear differential equation with polynomials as coefficients. Let $n(r, w-c)$ be the counting function of the zeros of the function $w-c(c=$ const $)$. Then

$$
L=\lim \sup _{r \rightarrow \infty} n(r, w-c) / r^{\rho} \leq \sigma \rho .
$$

In [42] the author considers the equation

$$
\mathrm{p}_{0}(z) \mathrm{w}^{\prime \prime}+\mathrm{p}_{1}(z) \mathrm{w}^{\prime}+\mathrm{p}_{2}(z) \mathrm{w}=0 \text {, }
$$

where $p_{0}(z) \neq 0, p_{1}(z)$ and $p_{2}(z)$ are entire and have real Taylor coefficients about any real point.

THEOREM 2.22. (Lopusans'kif [42]). Oscillatory real solutions of (2.10) have 
on1y real zeros.

THEOREM 2.23. (Lopusans'kii [42]). Solutions of (2.10) are oscillatory if and only if the function $\phi(z)=w(z) / \bar{w}(z)$ maps the upper half-plane conformally onto the unit disk, where $w(z)=w_{1}(z)+i w_{2}(z)$ and $w_{j}(z)(j=1,2)$ are two indenpendent real solutions of $(2.10)$, and their Wronskian is positive on the real axis.

The following characterization of the class $\mathrm{HB}$ (Hermite-Biehler) of entire functions having all their zeros within the upper half-plane is given in

THEOREM 2.24. (Lopušans'kii [42]). An entire function $F(z)$ is of class HB if and only if on the real axis it is a complex solution of an oscillatory equation of the form (2.10).

The ODE $w^{(n)}(z)+p_{n-2}(z) w^{(n-2)}(z)+\ldots+p_{0}(z) w(z)=\lambda^{n} w(z)$ is studied in [43], where $p_{0}(z), \ldots, p_{n-2}(z)$ are polynomials of degrees $m_{0}, \ldots, m_{n-2}$, respectively, and $\lambda$ is a complex parameter. It is proved that the fundamental system of solutions of the equation, determined by the identity matrix as initial conditions at $z=0$, satisifies the estimates

$$
\left|w_{i}(z, \lambda)\right| \leq|\lambda|^{c|z|^{\rho}} \operatorname{expc}|\lambda z|,
$$

for all sufficiently large values of $|\lambda|$ and $|z|$. The value of $\rho$ is defined by

$$
\rho=\max _{u \leq 1 \leq n-2}\left(m_{i}-i+n\right) /(n-i)
$$

and $c$ is some positive constant.

Asymptotic properties of the solutions of linear ODE with entire coefficients are studied in [44]. Consider the equation

$$
w^{(n)}+a_{n-1} w^{(n-1)}+\ldots+a_{0} w=0,
$$

where all the coefficients $a_{i}=a_{i}(z)(i=0,1, \ldots, n-1)$ are entire functions. Let $f(z)$ be a meromorphic function in the $z$-plane. Denote: $L(r, a, f)=$ $\max |z|=r^{2} n^{+}\left(|f(z)-a|^{-1}\right)$ if $a \neq \infty, L(r, a, f)=\max |z|=r^{+}|f(z)|$ if $a=\infty$. The function $\beta(a, f)$ is defined as

$$
\beta(a, f)=\lim \inf _{r \rightarrow \infty} L(r, a, f) / T(r, f),
$$

$T(r, f)$ is the usual Nevanlinna characteristic function of $f$. The authors call a solution $w(z)$ of $(2.11)$ a standard solution if $\beta(a, f)=0$ for all complex $a \neq 0, \infty$. 
THEOREM 2.25. (Boiko and Petrenko [44]). Each fundamental system of solutions of Eq. (2.11) contains at least one standard solution.

In [45] the author considers the first Painlevé equation $w^{\prime \prime}=6 w^{2}+z$ whose solutions are meromorphic of the form $w=1 /\left(z-z_{0}\right)^{2}-\left(z_{0} / 10\right)\left(z-z_{0}\right)^{2}-$ $\frac{1}{6}\left(z-z_{0}\right)^{3}+\ldots=1 /\left(z-z_{0}\right)^{2}-\phi^{\prime \prime}(z)$, where $\phi^{\prime \prime}(z)=-\sum_{n=0}^{\infty} \alpha_{n+2}\left(z-z_{0}\right)^{n}$.

She represents $w$ as a quotient of two entire functions:

$$
w=\left(u^{\prime 2}-u u^{\prime \prime}\right) / u^{2},
$$

where $u=\exp \left(-\int d z \int w d z\right)$, and then obtains recursion relations for the coefficients of the power series expansions of the numerator and denominator.

In conclusion, we note that in some recent works [46-50] entire solutions to $\mathrm{DE}$ of infinite order are discussed as well as properties of differential operators in spaces of entire functions. In [46] the author studies the existence of a solution to the equation $\Sigma_{n=0}^{\infty} a_{n} w^{(n)}(z)=f(z)$ whose growth equals that of the righthand side, in the case when $f(z)$ belongs to the class $B_{\phi, \psi}$ of entire functions $g(z)$ such that $|g(x+i y)| \leq \operatorname{cexp}[\phi(x)+\psi(y)]$, for any $x, y$; here the functions $\phi(x)$, $\psi(y)$ satisfy Hölder conditions. Let $\Psi(z)$ be an entire function on $\phi$ of exponential type without multiple roots. Let $M_{\Psi}$ be the operator of convolution with $\Psi(\delta)$, where $\delta$ is a $\delta$-function. The following result is proved in [47].

THEOREM 2.26. (Napalkov [47]). Each entire solution w(z) of the equation ${ }_{\Psi^{k}}{ }^{w} \equiv 0$ is representable in the form $w(z)=z^{k-1} w_{1}(z)+\ldots+w_{k}(z)$, where $M_{\Psi w_{i}} \equiv 0(i=1, \ldots, k)$, if and only if $|\Psi(z)|+\left|\Psi^{(1)}(z)\right|>c_{1} e^{-c_{2}|z|}$ for all $z \varepsilon \phi$ with some constants $c_{1}, c_{2}>0$.

In [48] the author studies the operator

$$
\mathrm{L}_{\mathrm{p}} \mathrm{w}=\sum_{i=0}^{\infty} \mathrm{p}_{i}(\mathrm{z}) \mathrm{w}^{(i)}(\mathrm{z}), \mathrm{p}_{i}(\mathrm{z})=\sum_{\mathrm{k}=0}^{\mathrm{p}} \mathrm{a}_{i k} \mathrm{z}^{\mathrm{k}}, \mathrm{p} \geq 0 .
$$

The operator $\mathrm{L}_{\mathrm{p}}$ is said to be

(1) applicable to the set $H$ of entire functions at the point $z_{0}$ if the series $\sum_{i=0}^{\infty} \mathrm{P}_{i}\left(\mathrm{z}_{0}\right) \mathrm{w}^{(i)}\left(\mathrm{z}_{0}\right)$ converges for any function $w$ from $\mathrm{H}$;

(2) applicable to $H$ in the domain $|z|<\infty$ if $L_{p}$ is applicable to $H$ at any finite point; 
(3) strongly regularly applicable to $\mathrm{H}$ inside the domain $|z|<\infty$ if, for any $\mathrm{W} \varepsilon \mathrm{H}$ and $\mathrm{R}<\infty$,

$$
\sum_{i=0}^{\infty} M\left(p_{i}, R\right) M\left(w^{(i)}, R\right)<\infty,
$$

where $M(f, R)=\sup \{|f(z)|:|z| \leq R\}$.

Let $Q$ be a bounded simply connected region. In this remarkable paper the author gives necessary and sufficient conditions for $L_{p}$ to be applicable to the set $R(Q)$ of exponential functions whose Borel transforms are regular on $C(Q)$. He proves that if the operator $L_{p}$ is applicable to $R(Q)$ at $p+1$ distinct points then

$$
\sup _{0 \leq k \leq p} \lim _{n \rightarrow \infty}\left|a_{n k}\right|^{1 / n} \leq 1 / \alpha \text {, }
$$

where $\alpha=\sup \{|z|: z \varepsilon Q\}$. Conversely, if (2.12) holds, then $L_{p}$ is strongly regularly applicable to $R(Q)$ inside $|z|<\infty$, and maps $R(Q)$ into itself.

In [49] the authors investigate the solvability of a class of functional equations, containing as a particular case differential equations of finite and of infinite order with constant coefficients, in the Banach space with weight of entire functions

$$
B_{\Phi(x, y)}=\left\{w(z) \varepsilon A_{\infty}:\|w\|=\sup _{z=x+i y \varepsilon}|w(z)| \exp (-\Phi(x, y))<\infty\right\} .
$$

Here $\Phi(x, y)$ is a locally bounded function in $R^{2}$ with a certain growth for $|z| \rightarrow \infty$.

The author [50] treats an equation $L w=f$ with $L=\sum_{i>0} P_{i}(z) d^{i} / d z^{i}$, where the $p_{i}(z)$ are polynomials, deg $p_{i}=n_{i}, \lim \sup \left(n_{i} / i\right)<\overline{1}$, in a space $[\rho, g(\theta)]$ of all entire functions satisfying $\lim \sup _{\mathrm{r} \rightarrow \infty}\left(\ln \left|\mathrm{w}\left(\mathrm{re}^{1 \theta}\right)\right| / \mathrm{r} \rho\right) \leq \mathrm{g}(\theta)$. Here $\mathrm{g}(\theta)$ is a trigonometrically $\rho$-convex function, $\rho>0$. It is proved that $L$ is a Noetherian operator, its index is found and the space of solutions of the corresponding homogeneous equation is investigated.

\section{DISTRIBUTIONAL AND ENTIRE SOLUTIONS OF FDE}

Finite order distributional solutions (2.1) of linear FDE have been studied in [15] and [51].

THEOREM 3.1. (Wiener [15]). The criterion for the existence of solutions (2.1) to the system 


$$
\operatorname{tx}^{\prime}(t)=\sum_{i=0}^{n} A_{i}(t) x\left(\lambda_{i} t\right)
$$

with matrices $A_{i}(t) \varepsilon C^{m}$ in a neighborhood of $t=0$ and constants $\lambda_{i} \neq 0$ is that some roots $\mu$ of the equation

$$
\operatorname{det}\left(\sum_{i=0}^{n}\left|\lambda_{i}\right|^{-1} \lambda_{i}^{-\mu} A_{i}(0)+(\mu+1) E\right)=0
$$

be nonpositive integers. If $\mathrm{m}$ is the smallest of their absolute values there exists a solution of order $\mathrm{m}$.

From here it follows that the system

$$
t x^{\prime}(t)=A(t) x(t)+\sum_{i=1}^{n} A_{i}(t) x\left(\lambda_{i} t\right)
$$

has a solution of order $m$ with support $t=0$, if $A_{i}(0)=0(i \geq 1)$ and $m+1$ is the smallest modulus of the negative integer eigenvalues of the matrix $A(0)$. This and similar results were used in [15] to investigate finite order solutions of some important equations of mathematical physics. For equations with more general argument delays we have

THEOREM 3.2. (Wiener [15]). The system

$$
t x^{\prime}(t)=\sum_{i=0}^{n} A_{i}(t) x\left(\phi_{i}(t)\right),
$$

in which $A_{i}(t) \varepsilon C^{m}, \phi_{i}(t) \varepsilon C^{1}$, has a solution (2.1) or order $m$, if the following hypotheses are satisfied: (1) the real zeros $t_{i j}$ of the functions $\phi_{i}(t)$ are simple and form a finite or countable set; (2) $A^{(k)}\left(t_{i j}\right)=0(k=0, \ldots, m)$, for $t_{i j} \neq 0$; (3) $\mathrm{m}$ is the smallest modulus of the nonpositive integer roots of Eq. (3.1) with $\lambda_{i}=\phi_{i}^{\prime}(0)$.

In [52] it was shown that, under certain conditions, the system

$$
x^{\prime}(t)=\sum_{n=0}^{\infty} A_{i}(t) x\left(\lambda_{i} t\right)
$$

has a solution

$$
x(t)=\sum_{n=0}^{\infty} x_{n} \delta^{(n)}(t)
$$

in the generalized-function space $\left(s_{0}^{\beta}\right)^{\prime}$ conjugate to the space $s_{0}^{\beta}$ of testing functions 
$\phi(t)$ that satisfy the restriction [2]

$$
\left|\phi^{(n)}(t)\right| \leq \operatorname{ac}^{n} n^{n \beta}, \beta>1 .
$$

To ensure the convergence of series (3.2), it is sufficient to require that for $n \rightarrow \infty$ the vectors $x_{n}$ satisfy the inequalities

$$
\left\|x_{n}\right\| \leq b d^{n} n^{-n \rho}, \rho>1
$$

since

$$
\begin{aligned}
& \left\|\sum_{n=0}^{\infty}<x_{n} \delta^{(n)}(t), \phi(t)>\right\|=\left\|\sum_{n=0}^{\infty}(-1)^{n_{\phi}(n)}(0) x_{n}\right\| \leq \\
& \quad \leq \sum_{n=0}^{\infty}\left|\phi^{(n)}(0)\right|\left\|x_{n}\right\| \leq \sum_{n=0}^{\infty}\left(c d n^{\beta-\rho)}\right)^{n}<\infty,
\end{aligned}
$$

for $\beta<\rho$. If series (3.2) converges, its sum rebresents the general form of a linear functional in $\left(s_{0}^{\beta}\right)^{\prime}$ ' with the support $t=0[53]$. Solutions in $\left(s_{0}^{\beta}\right)^{\prime}$ of some linear ODE with polynomial coefficients were studied in [54], [55], [56]. The particular importance of the system

$$
\sum_{i=0}^{\infty} \sum_{j=0}^{m}\left(A_{i j}+t B_{i j}\right) x^{(j)}\left(\lambda_{i} t\right)=t x(\lambda t)
$$

which was considered in [15] is that depending on the coefficients it combines either equations with a singular or regular point at $t=0$ and in both cases there exists a solution of the form (3.2). The equation

$$
t x^{\prime}(t)=A x(t)+t B x(\lambda t)
$$

provides an interesting example of a system that may have two essentially different solutions in $\left(s_{0}^{\beta}\right)^{\prime}$ concentrated on $t=0$. If the matrix $A$ assumes negative integer eigenvalues, (3.3) has a finite order solution (2.1). At the same time there exists an infinite order solution (3.2), if $A \neq-n E$ for all $n \geq 1$. In [3], [16], [57], and [58] the foregoing conclusions were extended to comprehensive systems of any order with countable sets of variable argument deviations. The basic ideas in the method of proof are applied to investigate entire solutions of linear FDE.

THEOREM 3.3. (Cooke and Wiener [3]). Let the system

$$
\sum_{i} \sum_{j=0}^{m} A_{i j}(t) x^{(j)}\left(\lambda_{i j}(t)\right)=0
$$


with a finite number of argument deviations, in which $x$ is an $r$-vector and $A_{i j}$ are $r \times r$ - matrices, satisfy the following hypotheses.

(i) The coefficients $A_{i j}(t)$ are polynomials in $t$ of degree not exceeding $p$ :

$$
A_{i j}(t)=\sum_{k=0}^{p} A_{i j k} t^{k}, A_{00}(t)=A t^{p}, p \geq 1
$$

(ii) The real-valued functions $\lambda_{i j}(t) \varepsilon c^{1}$ in a neighborhood of the origin, $\lambda_{i j}(0)=0$ and

$$
0<\left|\alpha_{00}\right|<1,\left|\alpha_{i j}\right| \geq 1, i+j \geq 1, \alpha_{i j}=\lambda_{i j}^{\prime}(0) \text {. }
$$

(iii) The matrix $A$ is nonsingular and

$$
c=\left|\alpha_{00}\right|^{-p-1}\|A\|-\sum_{i \geq 1}\left|\alpha_{i o}\right|^{-p-1}\left\|A_{i o p}\right\|>0 .
$$

Then in the space of generalized functions $\left(S_{0}^{\beta}\right)^{\prime}$ with arbitrary $\beta>1$ there exists a solution $x(t)$ supported on $t=0$.

In [3] it is also proved that system (3.4) with a countable set of argument deviations has a solution (3.2) if, in addition to the conditions of Th. 3.3, there exists a neighborhood of the origin in which each function $\lambda_{i j}(t)$ has the only zero $t=0$ and the series $\sum_{i=1}^{\infty} \alpha_{i}^{-1} A_{i}$ converges, where

$$
A_{i}=\max _{j, k}\left\|A_{i j k}\right\|, \alpha_{i}=\inf _{j}\left|\alpha_{i j}\right|, i+j \geq 1 .
$$

The choice of the coefficients in (3.4) enables us to consider both equations with a singular or regular point and to show that distributional solutions of FDE may be originated by deviations of the argument. The authors of [3] also investigate the system

$$
t^{P} x^{\prime}(t)=\sum_{i=0}^{\infty} \sum_{j=0}^{m} A_{i j}(t) x^{(j)}\left(\lambda_{i j}(t)\right)
$$

the particular cases of which

$$
t^{p} x^{\prime}(t)=A(t) x(t)
$$

and

$$
t^{p} x^{\prime}(t)=\sum_{i=0}^{\infty} A_{i}(t) x\left(\lambda_{i} t\right)
$$

have been studied in [56] and [57], respectively.

THEOREM 3.4. (Cooke and Wiener [3]). Suppose that system (3.5), in which $x$ is 
an $r$-vector and $A_{1 j}$ are $r \times r$-matrices, satisfies the following conditions.

(i) The $A_{i j}(t)$ are polynomials in $t$ of degree not exceeding $p+j-2$ :

$$
A_{i j}(t)=\sum_{k=0}^{p+j-2} A_{i j k} t^{k}, p \geq 2 .
$$

(ii) There exists a neighborhood of the origin in which the real-valued functions $\lambda_{i j} \varepsilon C^{1}$ have the only zero $t=0$ and $\left|\alpha_{i 0}\right| \geq 1$, inf $\left|\alpha_{i j}\right|>1 ;$ for $i \geq 0, j \geq 1$, $\alpha_{i j}=\lambda_{i j}^{\prime}(0)$.

(iii) The series $\sum_{i=0}^{\infty} \alpha_{i}^{-1} A_{i}$ converges, where

$$
\alpha_{i}=\underset{j}{\inf }\left|\alpha_{i j}\right|, A_{i}=\underset{j, k}{\max }\left\|A_{i j k}\right\| \text {. }
$$

Then there is a solution of (3.5) in $\left(s_{0}^{\beta}\right)^{\prime}$ with some $\beta>1$ supported on $t=0$.

The deep study of narrow classes of FDE, and even individual FDE, continues to remain one of the main problems. First of all, such equations can have some special, for example applied, interest. In addition, we can work out on them in the first instance methods of studying properties that are similar to properties of equations without deviation of the argument and are essentially new for equations with deviation, and then try to extend these methods, and the results obtained, to a broader class of FDE. In a number of papers [60-66] various authors have continued the study (originated in [59]) of the solutions, especially their asymptotic behavior as $t \rightarrow 0$ or $t \rightarrow \infty$, of the equation $x^{\prime}(t)=a x(\lambda t)+b x(t)$, which arises in certain technical problems, and also of systems and some more general equations of similar form. These works concern principally real solutions.

The author of [67] attacks complicated equations with elegant analytical tools. He investigates analytic solutions of the $\mathrm{FDE}$

$$
\sum_{j=0}^{r} \sum_{k=0}^{s} a_{j k} w^{(j)}\left(z^{k}\right)=0,0<q<1,
$$

with constant coefficients $a_{j k}$. Its formal solutions are obtained in the form of Mellin or Laplace integrals. The functions occuring in the integrands satisfy linear difference equations of the form $\Sigma_{V=0}^{n} P_{V}\left(q^{t}\right) G(t+v)=0\left(P_{V}(y)\right.$ polynomials $)$. Properties of solutions of such difference equations, in particular the location of 
singularities and the asymptotic behavior for absolutely large values of $t$, are studied. Conditions are derived for formal solutions of Mellin integral type to be actual solutions and these are shown to be often expressible as power or Laurent series. Solutions of Laplace integral type are shown to be representable as Dirichlet series under certain conditions. Finally, questions as to when the line of convergence of the Dirichlet series is the natural boundary of the function represented are discussed. The author asserts that the methods used can be extended to the case when the coefficients $a_{j k}$ are polynomials in $z$, and to some more general equations.

In [68] the growth of entire solutions of the FDE

$$
\sum_{k=0}^{m} a_{k} D^{k} w\left(\lambda^{m-k} z\right)=0, \quad D=d / d z
$$

is estimated by means of a suitably constructed comparison function. Furthermore, an explicit representation of all entire solutions is given which in certain cases leads to conclusions concerning lorations and multiplicity of the zeros of particular solutions. Finally, the growth of the maximum and minimum modulus of the solutions is compared which implies an estimate of the number of zeros. The FDE

$$
L w(z) \equiv \sum_{k=0}^{m} a_{k} D^{k} w\left(\lambda^{m-k} z\right)=f(z),
$$

where $a_{k}$ are complex numbers, $\lambda$ is a fixed parameter, $0<\lambda<1$, and the unknown w and the right member $f$ are entire functions, is considered in [69]. Introducing a generating function

$$
G(z)=\sum_{n=0}^{\infty} G_{n} z^{n}, G_{n}=\lambda^{n(n-1) / 2} / n !,
$$

the author shows that the general solution of (3.6) for $f=0$ is given by

$$
w(z)=\frac{1}{2 \pi i} \int_{\Gamma} G(t z) \tilde{w}(t) d t,
$$

with $\tilde{w}(t)=q(t) / \Delta(t)$, where

$$
\Delta(t)=\sum_{k=0}^{m} a_{k} \lambda^{k(2 m-k-1) / 2} t^{k},
$$

$\mathrm{q}$ is a polynomial of degree $\leq \mathrm{m}-1$ and $\Gamma$ is a contour enclosing a11 the zeros of $\Delta$. 
A similar integral representation is given for a solution of (3.6) with $f \neq 0$ in terms of the generalized Borel transform

$$
\tilde{f}(t)=\sum_{n=0}^{\infty} f_{n} / G_{n} t^{n+1}
$$

when

$$
f(z)=\sum_{n=0}^{\infty} f_{n} z^{n}
$$

In [70] the author discusses the system $w^{\prime}(z)=A w(\lambda z), 0<\lambda<1$, where $A$ is a complex constant matrix. First, the form of all entire solutions is given. Subsequently, for $z \neq 0$ a special system of particular analytic solutions is constructed by means of which all other solutions may be represented. The asymptotic properties as $\mathbf{z} \rightarrow \infty$ of all solutions are investigated. Furthermore, it is shown that given a specific asymptotic behavior, there is one and only one solution which possesses that asymptotic behavior.

Given the equation

$$
w^{\prime \prime}(z)+\sum_{k=1}^{m} a_{k}(z) w^{\prime}\left(\lambda_{k} z\right)+\sum_{j=1}^{n} b_{j}(z) w\left(\mu_{j} z\right)=0,
$$

where $a_{k}(z), b_{j}(z)$ are entire functions of finite order $\leq \rho$ and the constants $\lambda_{k}, \mu_{j}$ are in the set $0<|z|<1$, the author [71] shows that any solution $w(z)$ is also of finite order $\leq \rho$. As a special case he discusses the equation $w^{\prime \prime}(z)+p(z) w(\lambda z)=0$ where $0<\lambda<1$ and $p(z)$ is an entire function of finite order taking real values on the real axis, and derives an estimate on the type of a solution w(z).

The author of [72] studies properties of solutions to equations of the form

$$
w^{\prime}(\lambda z)=\sum_{k=0}^{m} a_{k}(z) w^{k}(z)
$$

where $a_{k}(z)$ are entire functions such that $T\left(r, a_{k}\right)=o(T(r, w))$ as $r \rightarrow \infty$, and $\lambda$ is a complex number, $|\lambda|=\sigma$, where $T(r, w)$ is the Nevanlinna function. He establishes the following

THEOREM 3.5. (Mohon'ko [72]). Let $w(z)$ be an entire solution of (3.7). If $\mathrm{m}=1$, then $\mathrm{w}(\mathrm{z})$ is of zero order, and if $\mathrm{m}>1$ then $\ln \mathrm{T}(\mathrm{r}, \mathrm{w}) \sim(\operatorname{In} \mathrm{m} / \operatorname{In} \sigma) \ln \mathrm{r}$ as $r \rightarrow \infty$.

The problem 


$$
\begin{aligned}
\sum_{i=0}^{n} a_{i} w^{(i)}(z) & =\exp (\alpha z) \sum_{i=0}^{n} b_{i} w^{(i)}(\lambda z), \\
w^{(i)}(0) & =w_{i}, i=0, \ldots, n-1,
\end{aligned}
$$

in which $a_{i}, b_{i}, \alpha$ and $\lambda$ are complex numbers, has been studied with various assumptions concerning parameters [73-77]. It is proved in [75] that, if $|\lambda|=1$, $\lambda \neq 1$, and $\left|a_{n}\right|>\left|b_{n}\right|$, its solution is an entire function. If $|\lambda| \leq 1, \lambda \neq 1$, and $\|\mathrm{C}\|<1$, the solution of the matrix problem

$$
\mathrm{W}^{\prime}(z)=\mathrm{AW}(z)+\exp (\alpha z)\left[\mathrm{BW}(\lambda z)+\mathrm{CW}^{\prime}(\lambda z)\right], \mathrm{W}(0)=\mathrm{W}_{0}
$$

is an entire function of exponential type [76]. These results were extended to 1inear FDE with polynomial coefficients and countable sets of argument delays in [7], [3] and [58]. The method of proof employs the ideas developed in the theory of distributional solutions.

THEOREM 3.6. (Wiener[58]). Suppose the system

$$
\begin{aligned}
& W^{(p)}(z)=\sum_{i=0}^{\infty} \sum_{j=0}^{p} Q_{i j}(z) W^{(j)}\left(\lambda_{i j} z\right), \\
& W^{(j)}(0)=W_{j}, j=0, \ldots, p-1
\end{aligned}
$$

in which $O_{i j}$ and $W$ are $r \times r$ - matrices, satisfies the following conditions:

(i) $Q_{i j}(z)$ are polynomials of degree not exceeding $m$;

(ii) $\lambda_{i j}$ are complex numbers such that

$$
0<q_{1} \leq\left|\lambda_{i j}\right| \leq 1,(j=0, \ldots, p-1), 0<q_{2} \leq\left|\lambda_{i p}\right| \leq q_{3}<1 ;
$$

(iii) the series $\sum Q^{(i)}$ converges, where $Q^{(i)}=\underset{j, k}{\max }\left\|Q_{i j k}\right\|$ and $Q_{i j k}$ are the coefficients of $Q_{i j}(z)$, and $\sum_{i=0}^{\infty}\left\|Q_{i p}(0)\right\|<1$.

Then the problem has a unique holomorphic solution, which is an entire function of order not exceeding $m+p$.

THEOREM 3.7. (Cooke and Wiener [3]). If, in addition to the hypotheses of Th. 3.6 the parameters $\lambda_{i j}(0 \leq j \leq p-1)$ are separated from unity: $0<q_{1} \leq\left|\lambda_{i j}\right| \leq q_{4}<1$, the solution of (3.8) is an entire function of zero order. THEOREM 3.8. (Cooke and Wiener [3]). Under the assumptions of Th. 3.3 there exists a polynomial $Q(z)$ of degree $p-1$ such that the system

$$
\sum_{i=0}^{k} \sum_{j=0}^{m} A_{i j}(z) W^{(j)}\left(\alpha_{i j} z\right)=Q(z)
$$


with positive constants $\alpha_{i j}$ has a solution $W(z)$ regular at $z=\infty$, and $w\left(z^{-1}\right)$ is an entire function of zero order.

THEOREM 3.9. (Wiener [51]). The problem

$$
\begin{gathered}
W^{\prime}(z)=\sum_{i=0}^{\infty} A_{i}(z) W\left(z-a_{i}\right)+\sum_{i=0}^{\infty} B_{i}(z) W^{\prime}\left(z-b_{i}\right), \\
\lim _{\operatorname{Rez} z-\infty} W(z)=W_{0}
\end{gathered}
$$

with $\mathrm{r} \times \mathrm{r}$ - matrices $\mathrm{A}, \mathrm{B}, \mathrm{W}$ has a unique holomorphic solution which is an entire function if :

(i) $A_{i}(z)=\sum_{k=1}^{m} A_{i k} e^{k z}, B_{i}(z)=\sum_{k=0}^{m} B_{i k} e^{k z} ;$

(ii) $a_{i}, b_{i}$ are complex numbers such that

$$
0 \leq \operatorname{Rea}_{i} \leq M_{1}<\infty, 0<M_{2} \leq \operatorname{Reb}_{i} \leq M_{3}<\infty
$$

(iii) the series $\sum A^{(i)}$ and $\sum B^{(i)} e^{-R^{-R e b}}$ converge where $A^{(i)}=\max _{k}\left\|A_{i k}\right\|$,

$$
B^{(i)}=\max _{k}\left\|B_{i k}\right\| \text {, and } \sum_{i=0}^{\infty}\left\|B_{i}(0)\right\| e^{-R e b_{i}}<1 \text {. }
$$

The authors [78] propose a method for finding polynomial solutions of the linear neutral FDE

$$
x^{\prime}(t)=b x(t)+\sum_{i=1}^{n} a_{i} x^{\prime}\left(t-r_{i}\right),
$$

where $b, a_{i}$ and $r_{i}>0$ are given constants. Meromorphic solutions of a class of linear differential-difference equations with constant coefficients are investigated in [79]. Numerous examples of FDE admitting entire solutions may be found in [40] and $[80]$.

In conclusion, we mention papers [81] and [82], where singular integral equations have been studied in spaces of generalized functions. However, it should be noted that, perhaps, the first work of this kind was [83].

\section{REFERENCES}

1. Fricke, G.H.; Roy, Ranjan and Shah, S.M. Bounded index, entire solutions of ordinary differential equations and summability methods, Inter. J. Math. \& Math. Sci. $4(3),(1981), 417-434$. 
2. Gel'fand, I.M. and Shilov, G.E. Generalized Functions, Vo1. 2, Academic Press, New York, 1968.

3. Cooke, Kenneth and Wiener, Joseph. Distributional and analytic solutions of functional differential equations, J. Math. Anal. and Appl. (to appear).

4. Gel'fand, I.M. and Shilov, G.E. Generalized Functions, Vol. 1, Academic Press, New York, 1968.

5. Wiener, Joseph. A retarded type system with infinitely differentiable coefficients has solutions in generalized functions, Uspehi Mat. Nauk $31(5)$, (1976), 227-228.

6. Strelitz, Sh. Asymptotic Properties of Analytic Solutions of Differential Equations, Izdat. "Mintis", Vilnius, 1972.

7. Wiener, Joseph. Entire solutions of a linear functional differential system, Differencial 'nye Uravnenija 13(3), (1977), 552-555.

8. Golubev, V.V. Lectures on Analytic Theory of Differential Equations, GITTL, Moscow, 1950.

9. Izumi, S. On the theory of linear functional differential equations, Tôhoku Math. J. 30 (1929), 10-18.

10. Robinson, L.B. Une pseudo-fonction et I'équation d'Izumi, Bu11. Soc. Math. France, 64 (1936), 66-70.

11. Robinson, L.B. Complémente á une étude sur l'equation fonctionelle d'Izumi, Bull. Soc. Math. France, 64 (1936), 213-215.

12. Aliev, F.S. The general solution of Euler's equation in generalized functions, Dok1. Akad, Nauk AzSSR, 20 (1) (1964), 9-13.

13. Aliev, F.S. The fundamental system of solutions of Euler's equation in generalized functions, Vestn. Mosk. Gos. Univ. Ser. Mat. Mekh. $\underline{5}$ (1964), 7-14.

14. Wiener, Joseph. Solutions of linear systems in generalized functions, Differencial 'nye Uravnenija $11(6)(1975), 1128-1130$.

15. Wiener, Joseph. Generalized-function solutions of linear systems, J. Differ. Equat. $38(2),(1980), 301-315$.

16. Wiener, Joseph. Generalized-function solutions of differential and functional differential equations, J. Math. Anal. and App1. 88(1), (1982), 170-182.

17. Sapkarev, Ilija. Eine Bemerkung über Polynomlösungen der homogenen 1inearen Differentialgleichungen, Bu11. Soc. Math. Phys. Macédoine 26(1975/1976), 5-8(1977).

18. Lazov, Petar; Dimitrovski, Dragan. Conditions for the existence of a maximal number of polynomial solutions for nonlinear differential equations, Prirod.Math. Fak. Univ. Kiril i Metodij Skopje GodiSen Zb. 25/26 (1975/1976), 101-106.

19. Lazov, Petar; Dimitrovski, Dragan. Conditions for the extstence of the maximal number of polynomial solutions of algebraic differential equations, Differencial'nye Uravnenija 13(6), (1977), 1131-1134.

20. Prolisko, E.G. Polynomial solutions of an equation of Liénard type, Differential equations and their application, Dnepropetrovsk. Gos. Univ. (1976), 105-112. 
21. Pisarenok, V.P. The behavior of solutions of a class of first-order differential equations in the complex plane, Differencial'nye Uravnenija 17(5), (1981), 930-932.

22. Kečkić, Jovan. Additions to Kamke's treatise. VIII. On singular solutions of generalized Clairaut's equation. Univ. Beograd. Publ. Elektrotehn. Fak. Ser. Mat. Fiz. 577-598 (1977), 30-32.

23. Gromak, V.I. Algebraic solutions of the third Painlevé equation, Dok1. Akad. Nauk BSSR 23(6), (1979), 499-502.

24. Gromak, V.I.; Cedel'nik, V.V. Rational solutions of Painlevé's fifth equation, Vesci Akad. Navuk BSSR. Ser Fiz-Mat. Navuk 6 (1978), 114-115.

25. Nova G., Lucimar. Certain properties of complex differential equations with polynomial coefficients, Rev. Colombiana Mat. 12(1-2), (1978), 13-58.

26. Wittich, Hans. Neure Untersuchungen über Eindeutige Analytische Funktionen, Springer-Verlag, Berlin, 1955.

27. Macintyre, A.J. On Bloch's theorem. Math. Z. 44(1939), 536-540.

28. Wittich, Hans. Ganze transzendente Lösungen algebraischer Differentia1gleichungen. Gött Nachrichten, (1952), 277-288.

29. Pöschl, Klaus. Zur Frage des Maximalbetrages der Lösungen linearer Differentialgleichungen zweiter Ordnung mit Polynomkoeffizienten, Math. Ann. 125(1953), 344-349.

30. Hille, Einar. Ordinary Differential Equations in the Complex Domain, John Wiley \& Sons, New York, 1976 .

31. Frei, M. Über die Lösungen linearer Differentialgleichungen mit ganzen Funktionen als Koeffizienten, Comment. Math. Helv. 35(1961), 201-222.

32. Valiron, Georges. Analytic Functions (Russian translation), Moscow, 1957.

33. Zimogliad,v.v. On the order of growth of entire transcendental solutions of second-order algebraic differential equations, Mat. Sb. $85(127), \underline{2}(6),(1971)$, 286-302.

34. Shah, S.M. Entire solutions of linear differential equations and bounds for growth and index numbers, Proc. Royal Soc. of Edinburgh 94A(1983), (to appear).

35. Roy, Ranjan and Shah, S.M. Vector-valued entire functions satisfying a differential equation (to appear).

36. Shah, S.M. On real continuous solutions of algebraic difference equations, Bull. Amer. Math. Soc. $\underline{53}(1947), 548-558$.

37. Shah, S.M. On real continuous solutions of algebraic difference equations, II, Proc. Nat. Inst. Sci. India 16 (1950), 11-17.

38. Cooke, K.L. The rate of increase of real continuous solutions of certain algebraic functional equations, Trans. Amer. Math. Soc. 92 (1959), 106-124.

39. Cooke, K.L. The rate of increase of real continuous solutions of algebraic differential-difference equations of the first order, Pacific J. Math. 4 (1954), 483-501. 
40. Bellman, Richard; Cooke, Kenneth L. Differential-Difference Equations, Academic Press, New York, 1963.

41. Knab, Otto. Zur Werteverteilung der Lözungen 1inearer Differentialgleichungen, Complex Analysis Joensuu 1978 (Proc. Colloq. Univ. Joensuu, Joensuu, 1978), Lecture Notes in Math. 747, Springer, Berlin, 1979, 189-204.

42. Lopusans'kii, $0 . \mathrm{V}$. The connection between functions of class HB and the solutions of second order linear differential equations, Dopovidi Akad. Nauk Ukraïn. RSR Ser. A $(9)$, (1975), 783-785.

43. Tkacenko, V.A. The growth of the solutions of a linear differential equation with polynomial coefficients, Mat. Fiz. 1 Functional. Anal. Vyp. 3 (1972), 71-76.

44. Boiko, S.S.; Petrenko, V.P. Asymptotic properties of the solutions of 1 inear differential equations with entire coefficients, Differenclal'nye Uravnenija $\underline{14}(11),(1978), 1923-1934$.

45. Fil'Cakova, V.P. The representation of meromorphic solutions of Painlevé's equation by entire functions, Dopovidi Akad. Nauk Ukraïn RSR Ser. A, (1975), 208-211.

46. Kubrak, V.K. The growth of particular solutions of a differential equation of infinite order, Izv. Severo-Kavkaz. NauXn. Centra Vysక̌. Školy Ser. Estestv. Nauk 1 (1977), 5-7.

47. Napalkov, V.V. A property of the solutions of differential equations of infinite order with constant coefficients, Izv. Vyš̌. Učebn. Zaved. Matematika $\underline{7}(182),(1977), 61-65$.

48. Korobeinik, Ju. F. Criteria for the applicability of differential operators of infinite order to certain classes of exponential functions. Godišnik Viss. Tehn. UČebn. Zaved. Mat. 8 (1972), (3), 9-18, (1973).

49. Korobeinik, Ju. F.; Bogačev, V.A. The solvability of linear differential equations in weighted spaces of entire functions, Differencial'nye Uravnenija $13(12),(1977), 2158-2167$.

50. Epifanov, 0.V. A differential operator with polynomial coefficients in classes of entire functions with a given estimator of the indicator, Mat. Sb. (N.S.) $114(156),(1981), 85-109$.

51. Wiener, Joseph. Solutions of functional differential equations in generalized functions, Differencial'nye Uravnenija, Ryazan', 3, (1974), 27-33.

52. Wiener, Joseph. Existence of solutions of differential equations with deviating argument in the space of generalized functions, Sibirsk, Mat. Z. 6(1976), 1403-1405.

53. Mityagin, B.S. On the infinitely differentiable function with the given values of the derivatives at a point, Dokl. Akad. Nauk SSSR 138(2), (1961), 289-292.

54. Aliev, F.S. On the solutions in generalized functions of some ordinary differential equations with polynomial coefficients, Dok1. Akad. NaukSSSR 167(2), $(1966), 259-262$.

55. Aliev, F.S. On the solutions in generalized functions of ordinary differential equations with polynomial coefficients, Dok1. Akad. Nauk SSSR 169(5), (1966), 991-994. 
56. Aliev, F.S. On the solutions of certain systems of ordinary differential equations in the space of generalized functions, Vestn. Mosk. Gos. Univ. Ser. Mat. Mekh. $\underline{5}(1973), 3-10$.

57. Wiener, Joseph. Generalized-function solutions of differential equations with a countable number of argument deviations, Differencial'nye Uravnenija 14 (2), (1978), 355-358.

58. Wiener, Joseph. Distributional and entire solutions of linear functional differential equations, Internat. J. Math.\& Math. Sci $\underline{5}(4),-(1982), 729-736$.

59. Kato, T.; McLeod J.B. The functional differential equation $y^{\prime}(x)=a y(\lambda x)+b y(x)$, Bul1. Amer. Math. Soc. 77, (1971), 891-937.

60. McLeod, J.B. The functional-differential equation $y^{\prime}(x)=a y(\lambda x)+b y(x)$ and generalizations, Conference on the Theory of Ordinary and Partial Differential Equations (Univ. Dundee, Dundee, 1972), Lecture Notes in Math. 280, Springer, Berlin, 1972 .

61. Chambers, L.G. Some functional differential equations, Quart. App1. Math. 32 $(1974 / 75), 445-456$.

62. Carr, Jack; Dyson, Janet. The functional differential equation $y^{\prime}(x)=a y(\lambda x)$ tby $(x)$. Proc. Roy. Soc. Edinburgh Sect. A 74(1974/1975), 165-174(1.976).

63. Carr, Jack; Dyson, Janet. The matrix functional differential equation $y^{\prime}(x)=$ Ay $(\lambda x)+B y(x)$, Proc. Roy. Soc. Edinburgh Sect. A $75(1975 / 1976),(1), 5-22$.

64. Lim, Eng-Bin. Asymptotic behavior of solutions of the functional differential equation $x^{\prime}(t)=A x(\lambda t)+B x(t), \lambda>0$. J. Math. Anal. App1. $55(3),(1976), 794-806$.

65. Lim, Eng-Bin. Asymptotic bounds of solutions of the functional differential equation $x^{\prime}(t)=a x(\lambda t)+b x(t)+f(t), 0<\lambda<1$. SIAM J. Math. Anal. $9(5),(1978)$, 915-920.

66. Pandolfi, L. Some observations on the asymptotic behavior of the solutions of the equation $\dot{x}(t)=A(t) x(\lambda t)+B(t) x(t), \lambda>0$. J. Math. Anal. Appl. 67 (2), (1979), 483-489.

67. Hahn, Wolfgang. Über die Funktional-Differentialgleichung $f^{\prime}(z)=f(q z)$ und verwandte Funktionalgleichungen, Ann. Univ. Sci. Budapest. Eötvös Sect. Math. 16, (1973), 3-21, (1974).

68. Vog1, Fritz. Das Wachstum ganzen Lösungen gewisser linearer Funktional-Differentialgleichungen, Monatsh. Math. 86 (3), (1978/1979), 239-250.

69. Vog1, Fritz. Über eine Integraldarstellung der ganzen Lösungen der FunktionalDifferentialgleichungen $\Sigma_{k=0}^{m} a_{k} D^{k} y\left(\lambda^{m-k} z\right)=f(z)$, Demonstratio Math. 12 (3), (1979), 645-655.

70. Vog1. Fritz. Über ein System linearer Funktional-Differentialgleichungen, $\underline{z}$. Angew. Math. Mech. 60 (1), (1980), 7-17.

71. Bélair, Jacques. Sur une équation differentielle fonctionnelle analytique, Canad. Math. Bull. 24(1), (1981), 43-46.

72. Mohon'ko, A.Z. Differential and functional equations with factors, Differencial'nye Uravnenija 15(9), (1979), 1713-1715. 73. Flamant, P. Sur une équation differentielle fonctionnelle linéaire, Rend. Circ. 
74. Bruwier, L. Sur l'application du calcul cymbolique á la iresolution d'equations fonctionneles, Bull. Soc. R. Sci. Liege 17, (1948), 220-245.

75. Valeev, K.G. On solutions of some functional equations, Iss1. po Integro-Diff. Uravn. v Kirgizii 5, (1968), 85-89.

76. Wiener, Joseph. Differential equations with periodic transformations of the argument, Izv. Vysš. Učebn. Zaved. Radiofizika 16 (3), (1973), 481-484.

77. Wiener, Joseph. Periodic maps in the study of functional differential equations, Differencial'nye Uravnenija, Ryazan', $\underline{3}(1974), 34-45$.

78. Fodcuk, V.I.; Holmatov, A. Polynomial solutions of differential-difference equations of neutral type. Approximate and Qualitative Methods in the Theory of Differential and Functional-Differential Equations, Akad. Nauk Ukrain. SSR, Inst. Mat., Kiev, 1979, 98-103.

79. Naftalevich, A.; Gylys, A. On meromorphic solutions of a linear differentialdifference equation with constant coefficients, Michigan Math. J. 27(2) (1980), 195-213.

80. Przeworska-Rolewicz, Danuta. Equations with Transformed Argument. An Algebraic Approach, PWN, Warszawa, 1973.

81. Kosulin, A.E. One-dimensional singular equations in generalized functions, Dokl. Akad. Nauk SSSR 163(5), (1965), 1054-1057.

82. Rogožin, V.S. A general theory of solving boundary problems in the space of generalized functions, Dokl. Akad. Nauk SSSR 164(2), (1965), 277-280.

83. Horváth, J. Sur 1'itération de la transformée de Hilbert d'une distribution complexe, C.R. Acad. Sci. 237(23), (1953), 1480-1482. 


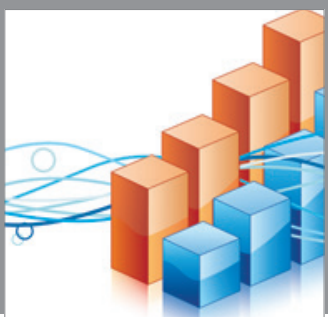

Advances in

Operations Research

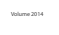

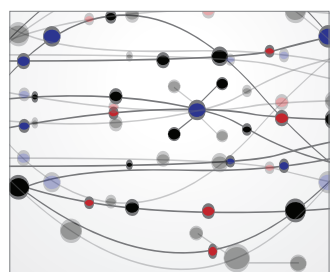

\section{The Scientific} World Journal
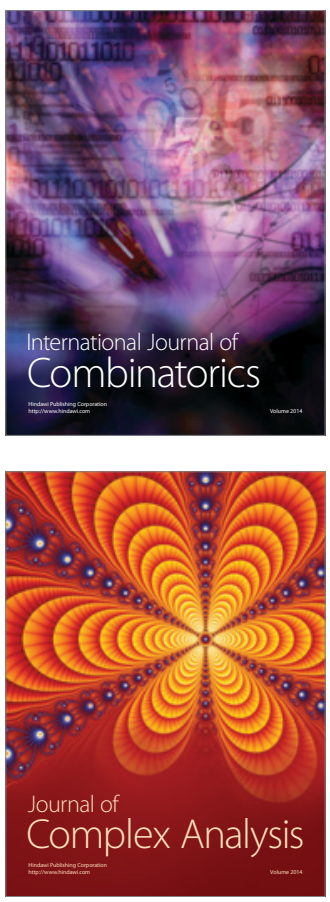

International Journal of

Mathematics and

Mathematical

Sciences
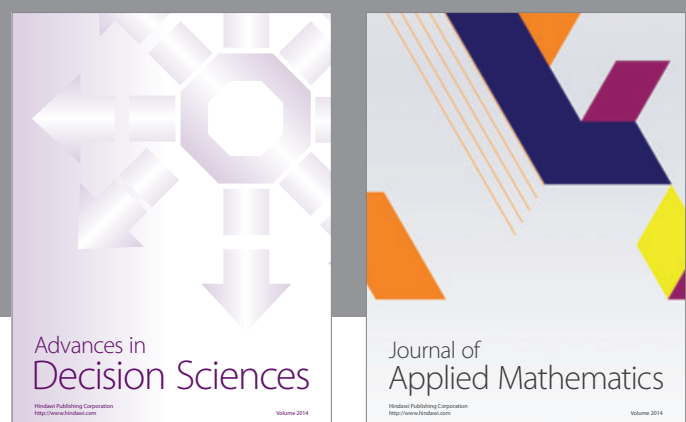

Journal of

Applied Mathematics
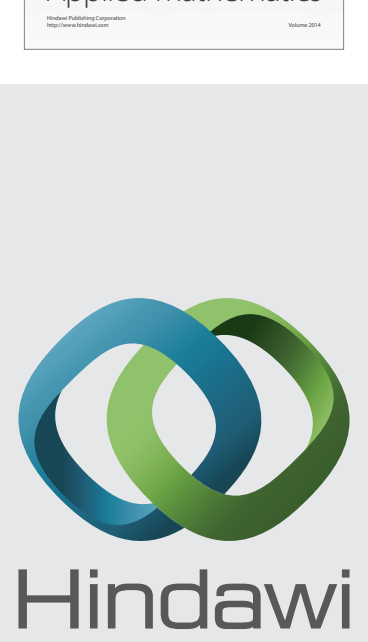

Submit your manuscripts at http://www.hindawi.com
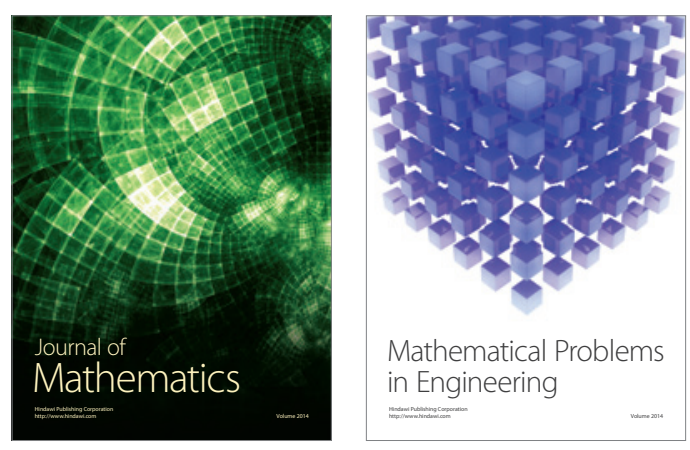

Mathematical Problems in Engineering
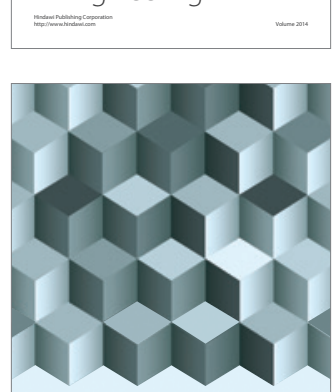

Journal of

Function Spaces
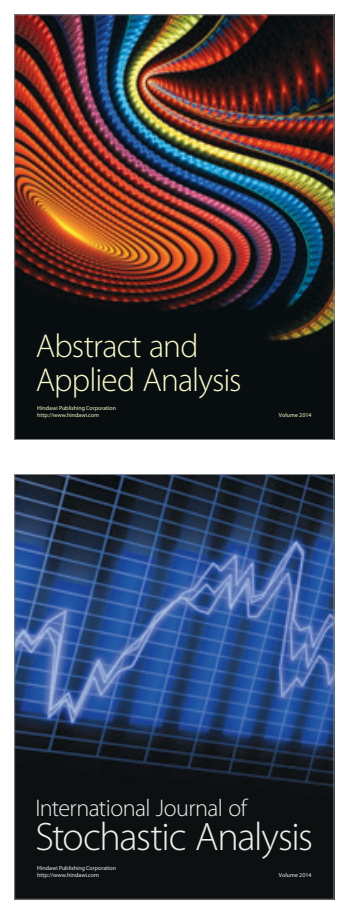

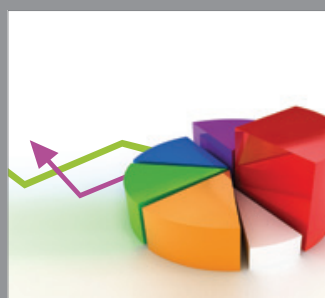

ournal of

Probability and Statistics

Promensencen
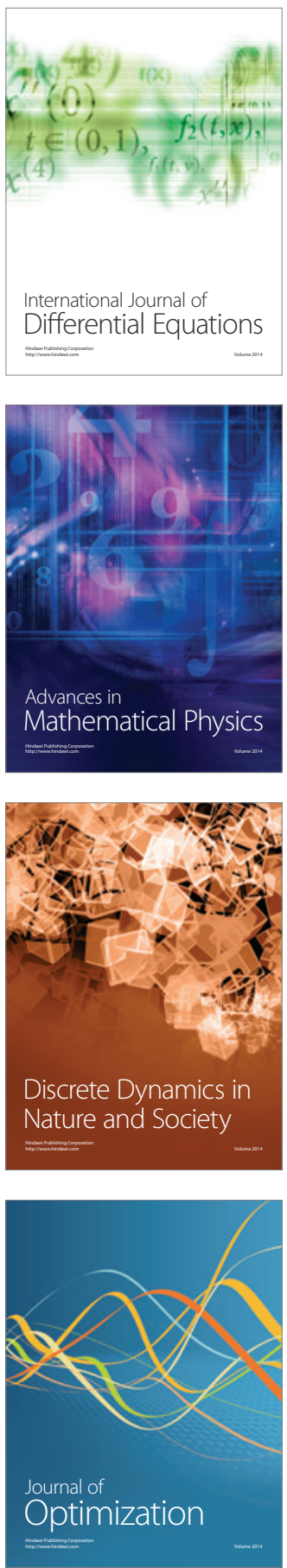